\title{
Servicios ecosistémicos y bienestar local: caso de estudio sobre productos de medicina natural en Panguipulli, sur de Chile
}

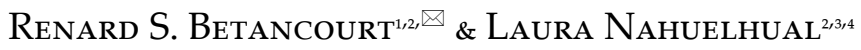 \\ ${ }^{1}$ Magister en Desarrollo a Escala Humana y Economía Ecológica, Facultad de Ciencias Económicas y Administrativas, \\ Universidad Austral de Chile. ${ }^{2}$ Instituto de Economía Agraria, Universidad Austral de Chile. Valdivia, Chile. ${ }^{3}$ Centro \\ FONDAP de Investigación en Dinámica de Ecosistemas Marinos de Altas Latitudes (IDEAL), Chile. ${ }^{4}$ Centro de Ciencia del \\ Clima y la Resiliencia (CR2), Chile.
}

Resumen. El presente estudio explora cómo se construye bienestar en comunidades locales del sur de Chile a través de prácticas sociales relacionadas con el uso de productos de medicina natural local (PMNL). Para la investigación se reconstruyeron historias de vida de actores sociales relevantes, por medio de entrevistas en profundidad. El análisis se basó en las teorías de la acción social y del desarrollo a escala humana, y sobre el enfoque de servicios ecosistémicos. Los resultados indican que en este territorio, el bienestar se construye socialmente a través de prácticas cotidianas dentro de la unidad familiar, en un ambiente mucho más privado (e.g., la transmisión de conocimiento entre padres e hijos al recolectar y reconocer plantas medicinales y su aplicación). Además, los actores sociales también construyen bienestar con prácticas comunitarias públicas, como es el caso de la mayoría de las iniciativas de recolección y procesamiento del Programa de Salud Inter-Cultural, que reponen el stock de medicinas. Por su parte, la teoría de la acción permitió observar prácticas sociales desde la vida cotidiana, mientras que el enfoque de servicios ecosistémicos ayudó a observar y a comprender los recursos naturales relacionados con estrategias de satisfacción local. Por esta razón, se convirtió en un anclaje teórico para comprender el bienestar desde la perspectiva del desarrollo humano. En este sentido, los principales satisfactores identificados fueron el Programa de Salud Inter-Cultural, la Mesa de Salud Inter-Cultural y los agentes culturales y unidad familiar. El Programa de Salud Inter-Cultural se relacionó de manera sinérgica con varias necesidades, mientras que la Mesa de Salud Inter-Cultural sólo se relacionó con las necesidades de entendimiento y participación. Finalmente, los agentes culturales y la unidad familiar se relacionaron con necesidades de afecto, ocio y libertad, entre otras.

[Palabras clave: bosques nativos templados, necesidades humanas, actores sociales, beneficios, satisfactores, redes sociales, conocimiento local]

\begin{abstract}
Aвstract. Ecosystem services and local wellbeing: Case study on natural medicine products in Panguipulli, southern Chile. This research explores how wellbeing is built through social practices related to the use of products of natural medicine (PNM) in local communities of southern Chile. Research inquiry involved the reconstruction of life-history of relevant local stakeholders, particularly through in-depth interviews (life-history method). The analysis relies on the social action theory, human scale development theory and the ecosystem services approach. Results indicate that, in this territory, wellbeing is socially constructed through everyday practices within the family unit, in a private atmosphere (e.g., the transmission of knowledge between parents and sons/daughters when they recollect, recognize and use medical herbs). Wellbeing is also built on another level, through social practices that are more public, such as the recollection and processing of medicine-plants carried out by the Intercultural Medicine Program. The social action theory allowed to observe social practices from everyday life, the ecosystem services approach helped to observe and understand natural resources, strictly related to local satisfaction strategies, becoming this way a theoretical anchor to understand well-being from the humane scale development perspective. In this sense, the main satisfactors identified were: the Inter-cultural Health Program, the Inter-Cultural Board, the cultural agents and the family unit. The Inter-Cultural Health Program is synergistically related to several needs, while the Inter-Cultural Board (discussion opportunity) is exclusively related to understanding and participation needs. Finally, the cultural agents and the family unit are related to the needs of affection, idleness and freedom, among others.
\end{abstract}

[Keywords: temperate native forest, human needs, social actors, benefits, satisfiers, social networks, local knowledge]

Editor asociado: Pedro Laterra

$\checkmark$ renard.betancourt.arellano@gmail.com
Recibido: 10 de marzo de 2016

Aceptado: 16 de diciembre de 2016 


\section{INTRODUCCIÓN}

La respuesta a la pregunta de qué se necesita para alcanzar bienestar difiere entre cada persona. Sin embargo, todos los seres humanos comparten necesidades fundamentales que deben satisfacer para poder experimentar bienestar (Max-Neef et al. 1993). El tema central de este estudio es entender la forma en que los servicios de la naturaleza contribuyen a la satisfacción de tales necesidades.

Desde 2005, y con la publicación de la Evaluación de Ecosistemas del Milenio (MEA 2005), la investigación en torno a los servicios de la naturaleza o servicios ecosistémicos (SE) ha aumentado de manera exponencial, incluyendo su identificación, cuantificación, mapeo, valoración económica y social, y el estudio de su gobernanza. No obstante, la pregunta sobre cómo categorizar y entender los constituyentes del bienestar a los cuales los SE contribuyen sigue estando poco respondida (Bennet et al. 2015). Lo anterior ha limitado la incorporación efectiva del enfoque de SE en la toma de decisiones en torno a la conservación de la naturaleza (Bennet et al. 2015; Guerry et al. 2015). Esto puede tener múltiples explicaciones. Una de ellas sería el hecho de que gran parte de la investigación en SE surge de la ecología y la economía, que no lograron profundizar en la relación entre SE y bienestar. Hasta el momento, en lo referente al análisis de los SE, los estudios sobre qué enfoques son más operativos para integrar el ejercicio interdisciplinario de la economía y ecología constituyen los pocos avances en la materia (Polasky and Segerson 2009).

A la fecha, la investigación dentro del enfoque de SE se centra en entender aspectos biofísicos en torno a la provisión de SE (Balvanera et al. 2012; Nahuelhual et al. 2015). Sin embargo, se ha indagado poco en los vínculos reales entre los seres humanos y los servicios de los ecosistemas que llevarían al mejoramiento del bienestar (Guerry et al. 2015). Los estudios que abordaron esta relación se centraron en cómo los SE contribuyen al alivio de la pobreza (Daw et al. 2011; Fisher et al. 2014; Sandhu and Sandhu 2014; Suich et al. 2015) o apuntan a entender valores sociales o socioculturales particulares (Iniesta-Arandia et al. 2014; Dawson and Martin 2015). Sin embargo, dicho enfoque va más allá que esto y, por el contrario, creemos que irrumpe como recurso retórico, incluso analítico, según las disciplinas y enfoques de quienes lo utilizan (Kull et al. 2015).
El enfoque de SE pretende vincular un determinado nivel de bienestar con lo que el individuo valora hacer y ser dentro de los límites y funciones de un ecosistema. Los SE, sustentados por funciones y procesos ecológicos, pueden ser capturados por las personas para generar bienestar. Inevitablemente, esto deja una huella en el paisaje (social y natural) y, por ende, es susceptible de ser observado (Ingold 1994; Miranda et al. 2015; Xu et al. 2016). No obstante, hoy en día es mucho más preciso hablar de coproducción entre ecosistemas y actividades humanas. Existen estudios que proponen reciprocidad entre humanos y ecosistemas bajo el concepto de "servicios a los ecosistemas" (Comberti et al. 2015). La lógica de este enfoque apunta hacia una relación de afectación mutua, cuyos resultados pueden variar desde la conservación, la extracción, degradación o depredación. De tal forma, se desecha la idea de una relación unidireccional entre SE y bienestar de las personas.

Para algunos autores, la rápida evolución de este enfoque revela nuevos retos para la ciencia básica, que ahora necesita evaluar, proyectar y gestionar SE junto con los cambios en el bienestar humano (Carpenter et al. 2009). Si el concepto de bienestar humano es complejo, controversial y en continua evolución (Fish et al. 2011), los vínculos entre SE y bienestar humano lo son aun más (Church et al. 2011). Y aunque algunos de estos se han identificado, otros permanecen poco estudiados y continúan siendo controversiales (Butler and Oluoch-Kosura 2006). En este sentido, explorar la diversidad de maneras en que las personas se relacionan a los SE frente a sus necesidades permite introducirnos en un espacio de investigación relacionado sólo con la diversidad y la diferencia cultural que, eventualmente, estructuran el bienestar.

Las distintas necesidades humanas requieren estrategias de satisfacción diversas que implican distintos vínculos con la naturaleza y que pueden (o no) corresponderse con una racionalidad económica. En este sentido, y tomando la discusión sobre ontologías de la naturaleza levantada por Escobar et al. (2011), se vuelve necesario entender el papel que juega la naturaleza para una comunidad, su economía y el potencial grado de bienestar que alcanza en relación a ella.

El objetivo de este estudio es analizar en profundidad las prácticas sociales (Bourdieu and Wacquant 2005) relacionadas con el uso de 
productos de medicina natural local (PMNL) y cómo ellas influencian la generación de bienestar local. Para ello se tomó como caso de estudio el Programa de medicina intercultural de la comuna de Panguipulli, en el sur de Chile. Además, se plantean las siguientes preguntas: ¿cuáles son los usos prevalentes de los PMNL en el área de estudio? ¿cuáles son las principales características de las prácticas sociales relacionadas a los PMNL? ¿qué elementos las diferencian? ¿qué necesidades humanas se pueden satisfacer con el uso de PMNL? ¿a qué componentes del bienestar, según MEA, se asocian esas necesidades humanas?

\section{Marco Teórico}

Paraentender prácticassocialesy construcción de bienestar, la sociología ofrece la teoría de la acción social, que propone la idea de habitus y campo social como herramientas analíticas de la práctica social (Bourdieu and Wacquant 2005). Dicho enfoque sugiere una sociología reflexiva, que entienda la complicidad ontológica (relación mutua fundamental) que hay entre las categorías mentales de las personas y las estructuras sociales que le dan vida a su realidad social local o, como definen más ampliamente los autores, al llamado campo social (e.g., el campo social del arte, de la academia, de la salud, etc.).

En este contexto surge el concepto de habitus, que se define como un conjunto de esquemas mentales, sentimentales, físicos o de prácticas, que permiten a la persona desarrollar percepciones, sensaciones, valoraciones, estructuras racionales, prácticas concretas y todo lo que eso implica en cuanto a generación y subordinación de normas sociales estructurantes de cierto orden social. En palabras de Bourdieu (1977), el habitus es "el principio generador de estrategias que permite a los agentes habérselas con situaciones imprevistas y continuamente cambiantes [...] un sistema de disposiciones duraderas y trasladables que, integrando experiencias pasadas, funciona en todo momento como una matriz de percepciones, apreciaciones y acciones y hace posible la realización de tareas infinitamente diversificadas" (Bourdieu 1977, pág. 214). Bajo esta lógica de análisis, la ontología de la naturaleza (i.e., la perspectiva humana con la que representa y ordena la realidad el sujeto) en este caso explica su relación con la naturaleza y da espacio para un tipo de habitus particular dentro de un campo social específico. En lo referente a las características genéricas del campo social, Bourdieu and Wacquant (2005) destacan lo siguiente: "el campo social prescribe sus valores particulares y posee sus propios principios reguladores. Estos principios delimitan un espacio socialmente estructurado en el que los agentes luchan, según la posición que ocupan en ese espacio, ya sea para cambiar o preservar sus fronteras y su forma."

Sin embargo, Bourdieu and Wacquant advierten que "(L)la relación entre el agente social (que encarna el habitus) y el mundo no es la que hay entre un sujeto (o una conciencia) y un objeto, sino una relación de complicidad ontológica o posesión mutua (...)". La complicidad ontológica que propone la sociología reflexiva a través de los conceptos de habitus y campo social se puede entender como 'espontaneidad imprecisa' del actuar 'natural' de las personas en sus realidades sociales. No obstante, esto también demuestra que la complicidad ontológica no es otra cosa que una forma de dominación, al ser una perspectiva fundamental de origen; ante cualquier diferencia se erige como un elemento de distinción y, por ende, como recurso y discurso político.

La sociología de Bourdieu explica la acción social desde un enfoque filosófico constructivista y estructuralista. Para ello elabora la idea de la lógica del sentido práctico, que tiene la acción social engendrada por el habitus que desarrolla un sujeto en un campo social. El sentido práctico es el que "preconoce; lee en el estado presente los posibles estados futuros de los que el campo [social] está cargado. Pues en el habitus, el pasado, presente y futuro se intersectan e invaden uno a otro" (Bourdieu 2005). En este sentido, la construcción de bienestar como práctica social es una medida de la participación del individuo en sociedad, práctica social instalada por una disposición a actuar en un campo social puntual. En otras palabras, el sentido de cualquier práctica queda cristalizado en la actividad misma. Estudiar esto nos permite aproximarnos a la realidad desde la práctica cotidiana.

Ahora es necesario definir cómo entendemos bienestar. Al usar el enfoque de SE nos circunscribimos al marco de MEA (2005), que entiende bienestar en base a cuatro constituyentes: a) seguridad, b) salud, c) bienes materiales básicos, y 4) buenas relaciones sociales. Estos cuatro constituyentes llevan hipotéticamente a una situación de libertad 
de acción que permite alcanzar lo que uno estima, en tanto ser y capacidades de hacer, de acuerdo al entorno cultural de origen. De tal forma, explorar cómo las comunidades locales construyen socialmente las estrategias para satisfacer sus necesidades a través del uso de un SE nos permitiría conocer tanto la capacidad explicativa del marco teórico que proponemos como algunos componentes estratégicos del campo social que se estudia, a saber: a) Estructura racional que accede al SE [1. Ontología: origen fundamental del pensamiento, límite racional, que establece el horizonte de posibilidades para entender y abordar la realidad; 2. Epistemología: manera y condiciones racionales de considerar 'válida' esa verdad particular; 3 . Práctica: actividad objetiva]; b) Estrategias prácticas de uso/ apropiación, disposición a actuar en términos sociológicos, y c) Satisfactores de construcción de bienestar.

Vistos desde la aproximación de los servicios de los ecosistemas, los PMNL combinan dimensiones de aprovisionamiento (e.g., consumo y uso directo del producto como medicina) y culturales (e.g., sistemas tradicionales de conocimiento de su uso), más prácticas humanas de producción o uso cotidiano (es decir sostenidas por una costumbre social). A través de los conceptos de habitus y campo social podremos interpretar los orígenes existenciales (ontológicos), las costumbres sociales (disposición a actuar) y las prácticas de uso, de la naturaleza en este caso, vinculadas a un territorio y a un campo social puntual (e.g., salud), muy ligado al paisaje y al ambiente. En la Figura 1 se representa cómo la complicidad ontológica opera a través del sujeto, sus orientaciones normativas y las estrategias que aportan a construir bienestar.
Finalmente, con el objeto de complementar la idea de bienestar propuesta por MEA (2005) utilizamos algunos conceptos de la teoría de desarrollo a escala humana. Dicha teoría rechaza la idea propuesta por la economía neoclásica, que considera a las necesidades humanas como infinitas e inclasificables. Por el contrario, la teoría de desarrollo a escala humana propone que las necesidades humanas son pocas, constantes entre las culturas y clasificables entre ellas (subsistencia, protección, afecto, entendimiento, participación, ocio, creación, identidad, libertad) (Max-Neef et al. 1993) (Figura 2). En cambio, bajo esa teoría, los satisfactores o estrategias para satisfacer las necesidades sí son infinitos y variables entre culturas. Entonces, satisfactor es la estrategia que permite alcanzar, de buena o mala manera, una determinada necesidad humana fundamental.

Bajo la teoría de desarrollo a escala humana, los satisfactores se dividen en cuatro dimensiones: ser (características), tener (herramientas), hacer (acciones) y estar (espacios). Cada una de ellas se cruza con las nueve necesidades humanas fundamentales para entregar satisfactores o estrategias de satisfacción de necesidades universales. Se reconocen distintos tipos de satisfactores según su modo de acción: a) sinérgicos, como el amamantamiento de un recién nacido (e.g., no sólo es positivo, sino también fundamental para su desarrollo como ser vivo, eventualmente ayuda a satisfacer todas las necesidades humanas desde la perspectiva del desarrollo a escala humana), b) singulares, que son aquellas estrategias particulares que satisfacen sólo una necesidad, c) inhibidores,

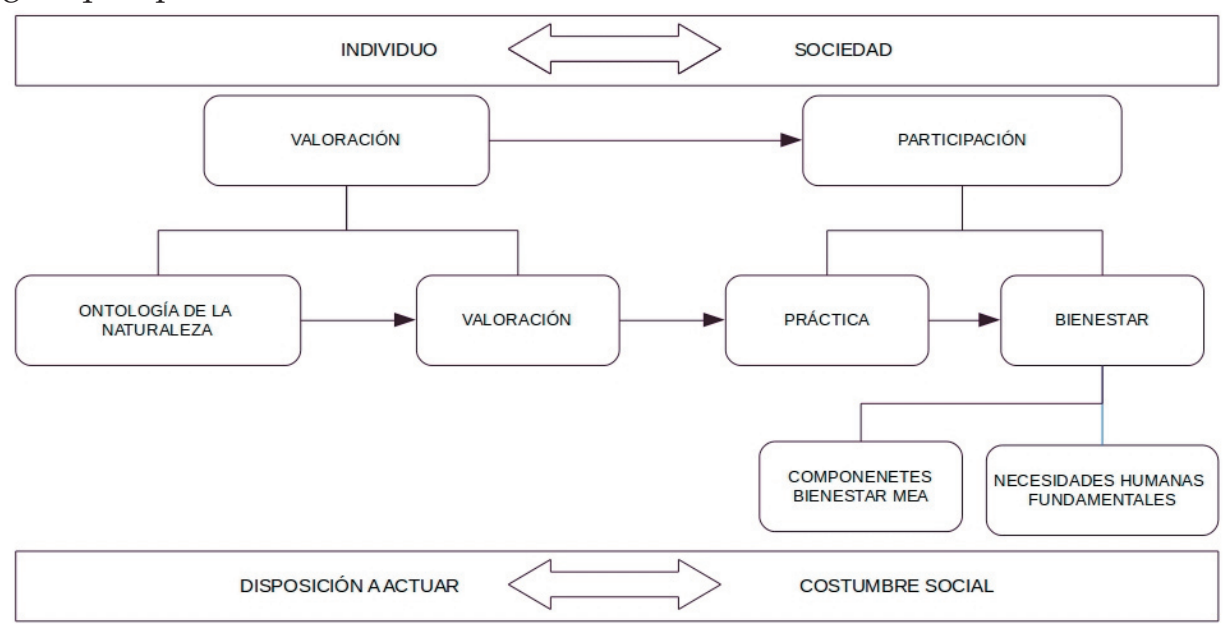

Figura 1. Esquema gráfico sobre construcción de bienestar y teoría de acción social.

Figure 1. Graphic representation of wellbeing construction and social action theory. 


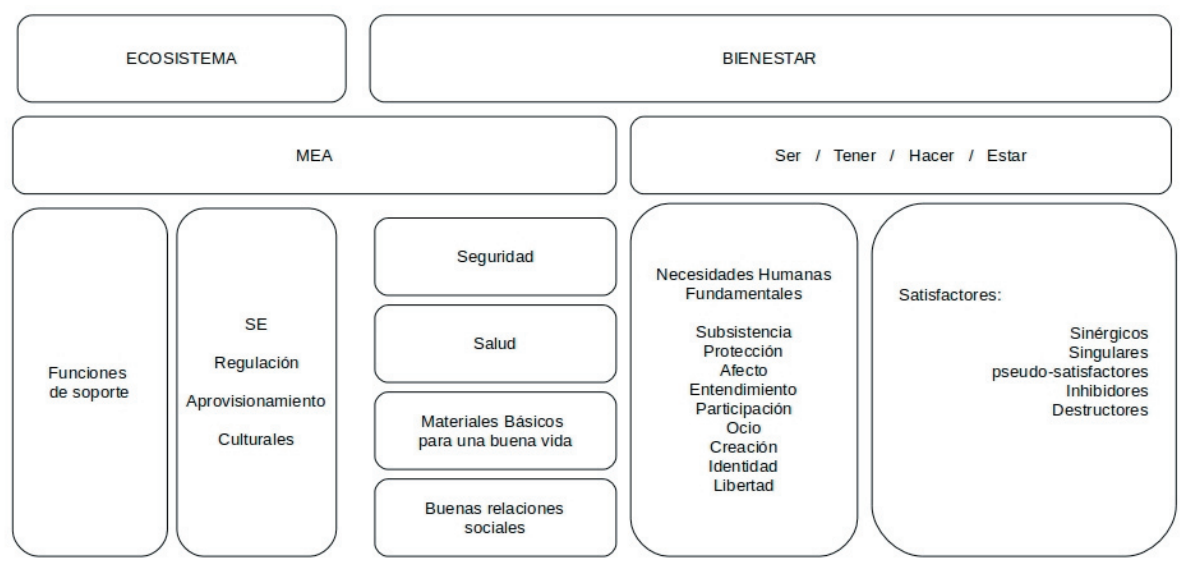

Figura 2. Extensión de bienestar según MEA (2005). Se le agrega la propuesta de necesidades humanas fundamentales de la teoría de desarrollo a escala humana.

Figure 2. Extension of wellbeing proposal according to MEA (2005), which is complemented by the human fundamental needs from the human scale theory.

que a pesar de satisfacer una necesidad complican la satisfacción de otras necesidades, d) pseudo-satisfactores, que aparentemente satisfacen, pero en realidad estimulan una falsa sensación, e) destructores, son aquellos que vuelven imposible satisfacer las necesidades humanas fundamentales (e.g., armamentismo, violencia, drogadicción, etc.).

\section{MATERIAles y MÉTOdos}

\section{Caso de estudio}

La comuna de Panguipulli $\left(39^{\circ} 38^{\prime} 0^{\prime \prime} \mathrm{S}\right.$ $72^{\circ} 20^{\prime} 0^{\prime \prime}$ O) tiene una superficie de $3292 \mathrm{~km}^{2}$ y una densidad poblacional de 9.1 habitantes/ $\mathrm{km}^{2}$. Se destaca por ser un territorio ancestral del pueblo mapuche. Algunos autores denominan a su sector pre-cordillerano como un área de refugio a la que accedieron las comunidades mapuches de la zona desde su desplazamiento a principios del siglo XX (Skewes et al. 2012). Según las cifras del censo 2002 (INE 2003), un tercio (10906 personas) de la población total (32718 personas) de la comuna pertenece a la etnia mapuche. Panguipulli, al igual que otras comunas con presencia indígena en el país, se encuentra ejecutando el Programa Especial de Salud y Pueblos Indígenas, que en este caso opera de manera formal en el Centro de Salud Familiar bajo el título resumido de Programa de Salud Inter-Cultural (en adelante el Programa). Este Programaesimplementado porelMinisteriode Salud de Chile, que en su reglamento orgánico establece la incorporación de un enfoque de salud intercultural en los programas de salud. Iniciativas como estas han sido ratificadas por Chile bajo el convenio 169 de la OIT para pueblos indígenas y tribales en el cual, entre otros puntos, se establece que "Los gobiernos deberán velar porque se pongan a disposición de los pueblos interesados servicios de salud adecuados o proporcionar a dichos pueblos los medios que les permitan organizar y prestar tales servicios bajo su propia responsabilidad y control, a fin de que puedan gozar del máximo nivel posible de salud física y mental" (Convenio 169 OIT, pág. 43).

El conocimiento y el uso de plantas medicinales del bosque nativo es parte central del pueblo mapuche (Sepúlveda 2006). También lo es su entorno natural, espacio ecológico para la práctica espiritual y medicinal (Neira et al. 2012). Por lo mismo, fue central aprobar el convenio 169 de la OIT. En consecuencia, el Programa está muy ligado al uso de los PMNL y a la medicina mapuche, componentes en los que se centra esta investigación.

\section{Tipo de investigación y unidades de análisis}

Esta investigación posee un carácter descriptivo y un enfoque cualitativoetnográfico. Bajo este enfoque se abordan realidades subjetivas llenas de "estructuras conceptuales complejas, muchas de ellas superpuestas o enlazadas entre sí, que son al mismo tiempo extrañas, irregulares, o explícitas (...)" (Geertz 1996). Por medio de este enfoque se buscó indagar en el cómo y los porqués de la relación con los PMNL de los entrevistados. Se usó para ello el método de historias de vida, que incluyó: a) un biograma [i.e., línea temporal biográfica que expresa los principales hitos a lo largo de la vida del entrevistado/a], b) una reminiscencia [i.e., 
ejercicio de asociación libre], y c) dos etapas de reflexión en profundidad. Este método permite entre otras cosas, desarrollar mayor profundidad en la indagación de los datos, al mismo tiempo que el entrevistador desarrolla mayor confianza con el entrevistado.

Todos los entrevistados reconstruyeron una línea cronológica de su vida (biograma) al asociar los hitos más importantes relativos al uso de PMNL a lo largo de su historia. Para construir su relato se apoyaron en sus valores, costumbres y/o hábitos cotidianos. Además, sobre la base de otro ejercicio de asociación libre se solicitó a cada entrevistado construir una historia o cuento sobre su relación con los PMNL, para lo cual debió vincular aspectos cotidianos u otros que lo marcaron en algún momento de su vida (reminiscencia). A través de las entrevistas en profundidad se solicitó a cada entrevistado reflexionar en dos etapas respecto a su historia de vida: a) niñez/ juventud, y b) adultez, todo en relación con el uso y sus percepciones sobre los PMNL. La pauta de entrevista en profundidad le sugería al entrevistado temas que estimulaban la reflexión en torno a las necesidades humanas fundamentales y a las dimensiones del ser, según la teoría de desarrollo a escala humana. Dichos temas se combinaban en la reflexión en profundidad con los temas previamente sugeridos por el mismo entrevistado en el biograma. Las personas entrevistadas podían iniciar cualquiera de las dos etapas reflexivas (niñez/adultez) cuando lo estimaran conveniente, tanto durante la realización del biograma o de la reminiscencia.

Se analizó el relato y las prácticas desde un lugar relevante en la estructura social, y se atendió especialmente el concepto de muestra estructural sugerido por Montañés (2013). Así, nos enfocamos en particular en las personas que trabajan en el Programa antes mencionado y en sus respectivas oficinas, ubicadas en algunos consultorios rurales en Panguipulli. Se realizaron en total 12 entrevistas y se utilizó el criterio de saturación de discurso para delimitar la cantidad de entrevistas. Nueve entrevistas fueron en profundidad e incluyeron el biograma, la reminiscencia y las reflexiones, y se aplicaron a agentes culturales, que aportaban conocimiento y trabajo al Programa, más tres entrevistas abiertas adicionales a informantes clave (i.e., la coordinadora general, asistente social y enfermera del Programa). El trabajo de terreno se realizó en varias jornadas entre los meses de abril y diciembre de 2015.

\section{Conceptos clave: lawen y agentes culturales}

En general, el concepto que los mapuches utilizan para referirse a la medicina o a la hierba es 'lawen', y cuando se refieren a una medicina que es clave y sagrada por ellos utilizan la palabra 'küme', que se podría traducir como 'buena' o 'sabia/sagrada'. En ese mismo sentido, también utilizan el concepto 'küme mongen' para hacer referencia a prácticas de vida ancestrales que expresan un buen vivir. Por su parte, el concepto que utilizan en el Programa para hacer referencia a aquellas personas que trabajan allí, pero que pertenecen a las comunidades mapuche y no a la institucionalidad formal del servicio de salud, es agentes culturales. Ellos portan un cargo en el campo de la salud dentro de sus comunidades y son muy respetados por ser cuidadores y guardianes tanto de las costumbres, conocimientos, como también de las plantas propiamente tal. Se pueden reconocer los siguientes agentes culturales: machi, lawentuchefe, huesero, partera, ñaña y recolectores/as. El/la machi, como curanderos de enfermedades/males en la cultura mapuche, siempre están asociados al mundo espiritual y de sanación general de las enfermedades. Este mundo está ligado a los espacios ecológicos y al entorno natural (Neira et al. 2012) por lo tanto es prácticamente la figura de mayor jerarquía tanto en el Programa como en la comunidad mapuche. El/la lawentuchefe son cuidadores de los lugares y de las plantas medicinales, y el huesero o componedor de huesos, la partera y los recolectores/as portan títulos que son auto explicativos. Finalmente, las ñañas son mujeres (madres o abuelas) que aportan al Programa como recolectoras o cuidadoras de saberes.

\section{Selección de los informantes y entrevistados}

Los tres informantes clave que seleccionamos pertenecían al Centro de Salud Familiar de Panguipulli, dedicados en jornada completa a coordinar tanto a los agentes culturales como a los pacientes que llegaban al Programa. Por lo tanto, eran fuente de información fundamental. Las historias de vida (nueve en total) se realizaron sólo a los agentes culturales por recomendación de los informantes clave. La razón que primó para elegir a estos agentes culturales fue que como ocupantes de sus respectivos cargos han estado expuestos/as desde edad temprana a la medicina tradicional mapuche. Entre los agentes culturales había hombres y mujeres en similares proporciones, 
todos/as mayores de 40 años y nacidos en sectores rurales del sur de Chile.

\section{Resultados}

\section{Práctica social: habitus y campo social de los} PMNL

Lashistorias devida (biograma, reminiscencia y entrevista en profundidad) mostraron que el habitus en el campo social de la salud está compuesto por una disposición a actuar típicamente mapuche. Es decir, para los mapuches, los PMNL y las personas que los conocen, son valiosos y hay que conservarlos porque mantienen la salud de las personas y del entorno. Al analizar la estructura racional que opera en el entrevistado, desde los relatos y desde las prácticas sociales relacionadas al uso de PMNL queda en evidencia una cultura local diferenciada en términos epistemológicos y ontológicos. Es decir, se diferencia en cuanto a cómo interpretan lo que es conocimiento 'válido' desde el origen racional fundamental, constituido por una manera de pensar y hacer distinto a lo tradicional científico. En particular, la diferencia con la cultura nomapuche (chilena) emerge desde la noción mapuche de que la salud depende del entorno natural, y se puede interpretar/consultar desde su relación con la naturaleza y, en ese sentido, con el propio espíritu.

"Los espiritus son caprichoso', si lo pillan (encuentran) a uno débil te invaden y te hacen hartos males. Hoy en dia hay hartas gentes enfermas, pero no sólo porque no se cuiden, sino porque tampoco se preocupan de la naturaleza y nosotros somos naturaleza y ya no quedan personas que sepan cuidarla. Los espíritus, los Ñgem, como decimos nosotros, muchos han muerto" (Machi, Panguipulli).

Los relatos evidencian percepciones de lo difícil que ha sido mantener la costumbre y rescatar algo del conocimiento de antaño sobre el uso de PMNL. En ese sentido, la práctica habitual y la disposición a actuar para producir, cuidar y ejercer la medicina mapuche reflejan una gran preocupación por rescatar actores clave de su propio campo. La disposición a actuar, las percepciones y las prácticas identificadas en las historias de vida sugieren costumbres que ellos consideran antiguas y nuevas. Las prácticas antiguas se relacionan con la atención de los pacientes y también con el proceso de recolección y conservación del lawen. Dentro de las prácticas que ellos consideran nuevas, se destaca el uso y la preparación de ungüentos, así como también la recolección y la conservación de hongos silvestres.

Los entrevistados estaban distribuidos territorialmente en sectores muy distantes del centro de la comuna, que es donde se encuentra el Centro de Salud Familiar. Sin embargo, existen pequeñas sedes, que se denominan postas rurales, repartidas por varios sectores de la comuna. En todas ellas se realizaban actividades de procesado y conservación de los PMNL, de mantención de los conocimientos y de fortalecimiento de las relaciones con las personas que los portan.

Las principales características del habitus es que busca rescatar conocimiento ancestral, técnicas de tratamiento y conservación de medicinas, todo desde la lógica de no división entre la salud, los espíritus y el medio ambiente. En este sentido, las percepciones identificadas en las historias de vida recorren gran parte del territorio (comuna de Panguipulli) y cada una de las localidades donde se ubican los agentes culturales (ciudades de Panguipulli, Pocura, Liquiñe y Neltume). Al mismo tiempo, clasificamos las percepciones en cuatro categorías básicas, a propósito de la saturación del discurso, que fueron emergiendo en el transcurso de las entrevistas (Tabla 1). Esto

Tabla 1. Percepciones de uso de PMNL identificadas en este estudio.

Table 1. Perceptions of use of PNM indentified in this study.

\begin{tabular}{|c|c|c|c|c|}
\hline $\begin{array}{l}\text { Territorios de la comuna } \\
\text { vinculados al Programa }\end{array}$ & $\begin{array}{l}\text { Económico } \\
(+)^{*}\end{array}$ & $\begin{array}{c}\text { Político } \\
(+)^{*}\end{array}$ & $\underset{(+)^{*}}{\text { Ambiental }}$ & $\begin{array}{l}\text { Cultural } \\
(-)^{*}\end{array}$ \\
\hline Panguipulli & Crematístico & Küme lawen & $\begin{array}{l}\text { Conservación y } \\
\text { protección }\end{array}$ & $\begin{array}{l}\text { Sabiduría ancestral } \\
\text { en rescate }\end{array}$ \\
\hline Pocura & Crematístico & $\begin{array}{c}\text { Küme lawen resignificación } \\
\text { de rol y estatus }\end{array}$ & $\begin{array}{l}\text { Conservación y } \\
\text { protección }\end{array}$ & $\begin{array}{c}\text { Sabiduría ancestral } \\
\text { en rescate }\end{array}$ \\
\hline Liquiñe & Crematístico & $\begin{array}{c}\text { Küme lawen resignificación } \\
\text { de rol y estatus }\end{array}$ & $\begin{array}{l}\text { Conservación y } \\
\text { protección }\end{array}$ & $\begin{array}{c}\text { Sabiduría ancestral } \\
\text { en rescate }\end{array}$ \\
\hline Neltume & Crematístico & Küme lawen & $\begin{array}{l}\text { Conservación y } \\
\text { protección }\end{array}$ & $\begin{array}{l}\text { Sabiduría ancestral } \\
\text { en rescate }\end{array}$ \\
\hline
\end{tabular}

* Percepción positiva (+): Percepción negativa (-) 
es una evidencia de cómo el habitus se emerge en el campo social de la salud y del medio ambiente. Para el Programa, el campo social de la salud incluye el entorno natural, las plantas y las personas; sin embargo, su habitus gravita más en función del entorno natural, razón por la cual las percepciones tienen mayor relación con el bosque, el monte, los ríos que con el propio cuerpo humano. Por otro lado, sobre la base humana e institucional que ha conseguido el equipo de trabajo del Programa es posible identificar una ruta principal por donde transita el SE a lo largo del territorio de Panguipulli, cuyo movimiento se sostiene por el trabajo y el conocimiento que algunas personas puntuales tienen sobre el territorio y sus componentes, como se explica en la siguiente sección.

\section{Estrategias de construcción de bienestar}

Coproducción e intercambio de los PMNL en el territorio. Los PMNL usados tienen un nivel de secreto o confidencialidad elevado, no sólo para el investigador (por ser totalmente ajeno a estas comunidades), sino también para cualquiera que no tenga el estatus de agente cultural. La razón es que el celo de robo de información y pérdida de lawen ya es muy normal para ellos, como queda reflejado en el siguiente testimonio de una ñaña (abuela) del sector Liquiñe.

"Por eso no nos gusta hablar de nuestro lawen. Ya no crecen donde antes siempre lo hacian. Además, si es que llegamos a hablar un poquito que sea, capaz que de nuevo venga una persona con título universitario o un afuerino y haga un negocio con las plantas a espaldas de nosotros ¿Usté cree que nosotros ganamos algo de eso?" (ñaña, sector Liquiñe)

Existen dos tipos de tránsito de SE. El primero se relaciona con la provisión directa hacia el Programa; el segundo, con iniciativas personales de los agentes culturales, que pueden tener características pecuniarias o de conservación de las plantas y del conocimiento. El tránsito de provisión de SE hacia el Programa va de manera directa desde un proveedor cercano a alguna de las postas rurales de atención hasta la red de asistencia central del Programa en Panguipulli. Este fenómeno ocurre a través de una asociación de iniciativas locales personales y colectivas, lo que implica identificar dónde hay "remedio" (i.e., la medicina) y quién sabe usarlo. En este sentido, la integración del SE para el pueblo mapuche sucede en hospitales.
"Hay un servicio que se entrega en el hospital, que es para las comunidades mapuche. Por ejemplo, si quieren que su bebé nazca bajo la tradición mapuche o por ejemplo si es el hijola de un longko (líder mapuche) y hay que darle una gota (de agua o extracto de lawen) de alguna cascada especial porque así manda la tradición, hay que ir a buscarla y se le da al recién nacido/a" (informante clave, sector Panguipulli).

El tránsito vinculado a los intereses más personales de los agentes culturales, en ocasiones implicaba el movimiento de los PMNL fuera de la red del Programa y fuera de la comuna

"Yo tengo una socia con la que hacemos negocios allá en Pucón (localidad fuera de la comuna de Panguipulli) y también nos ayudamos a cuidar plantas que han desaparecido. Yo llevo algunas plantas para allá para vender y también me traigo para acá alguna planta que ya no se ve mucho. Es para cuidar también" (lawentuchefe, sector Pocura).

"Tengo hongos acá en el terreno donde está mi casa y también allá en el otro terreno que tengo cerca de la reserva nacional (Mocho Choshuenco). A mí me sirve eso porque quiero aprender a conservarlos para vender, hacer negocio. Y bueno también porque esto seguro mis ancestros sabian identificarlos, yo siempre me interesé desde chico, esto es -como quién dice- sabiduría también pue'" (recolector, sector Punahue).

El tránsito del SE PMNL no sólo involucra el movimiento, también considera su dirección y su trayectoria. En ese sentido, la trayectoria va desde y hacia la naturaleza con una clara iniciativa de conservación, de forma tal que los PMNL se mantengan. La coproducción se sostiene a través de la casi exclusiva participación e integración de diferentes agentes culturales. Así, el SE (cantidad, calidad e intensidad) es de responsabilidad compartida tanto de agentes culturales como de profesionales de la salud dedicados al Programa.

"A mí (agente cultural) me invitó a participar en esto la señorita asistente social, bueno en verdad fue la persona que vino antes de ella, pero ella volvió a preguntarme. Yo dije que sí porque es importante participar de la Mesa (de trabajo, coordinación y decisión) y como yo soy conocida acá, por las tejedoras y eso, saben que me interesan las plantas" (lawentuchefe, sector Liquiñe). Cabe destacar que la mención a las tejedoras se refiere a un caso local de superación de un estado depresivo crónico, identificado 
en la posta rural de Liquiñe con un amplio conjunto de mujeres adultas, a través de la consolidación de un grupo de tejido con lana de oveja. Eventualmente, se convirtió en un emprendimiento local de destacado éxito en términos de salud mental y mejora económica.

Las entrevistas muestran que la combinación de esfuerzos permite que los equipos de salud del Programa consigan y distribuyan los PMNL a lo largo del tránsito principal identificado en el territorio. Los PMNL se obtienen en áreas de bosque nativo, y entre ellos se encuentran especies como Quinchamalium chilensis (quinchamali), Adesmia emarginata (paramela), Drimys winteri (canelo), Ribes punctatum (zarzaparrilla), Caldcluvia paniculata (tiaca), Gunnera tinctorea (nalca), Buddleja globosa (matico), Berberis spp. (michay), Weinmania trichosperma (tineo) y Aristotelia chilensis (maqui).

En cuanto a las percepciones sobre el uso de PMNL identificadas a través de las historias de vida, lo primero que cabe destacar es que, a propósito de la iniciativa del Programa, todos los entrevistados valoran de forma positiva rescatar la sabiduría ancestral ya que es un conocimiento que les reafirma su identidad mapuche y los invita a participar de una actividad comunitaria o pública, que en principio beneficiará a todo el territorio. En este sentido, el tránsito principal del SE PMNL es acompañado en parte por esta percepción positiva de uso del lawen, pero no se pierde la posibilidad de generar alguna ganancia vendiendo o transando PMNL. Sin embargo, la motivación fundamental es participar de esta iniciativa que les permitiría vivir en equilibrio con una buena salud, como lo expresan los siguientes extractos de los relatos.

"Yo le aporto a ella (coordinadora del Programa) con hierbitas y cosas cuando me pide, porque piense usted que los remedios (hierba) se están corriendo, están desapareciendo. Y aquí atienden con lawentuchefe, machi y to'a la custión (todo lo demás). Entonces hay que aportar pue', si uno las tiene..." (ñaña y vendedora oficial en mercado Trafkintuwe de Panguipulli, sector Pullingue).

La idea de aportar, de apoyarse unos con otros, traspasa sus respectivas funciones o trabajos ante la necesidad común del territorio de recolectar no sólo plantas, sino también conocimiento sobre su uso y aplicación. La dimensión humana opera como canal de apoyo y transmisión tanto de plantas difíciles de conseguir como de personas que saben de medicina mapuche. Al mismo tiempo, la percepción positiva viene de la mano con un discurso político muy arraigado en la tradición mapuche.

El concepto küme mongen, de vivir bien o buen vivir, en equilibrio con el ambiente, contiene la idea del küme lawen (vocablo en lengua mapuche que significa medicina o hierba-sabia, buena), es decir, de una medicina equilibrada que se mantiene a lo largo de la vida a través de la rutina. Sin embargo, este discurso se ve contrastado con otra situación. Panguipulli no está ajeno a los grandes procesos de modernización y globalización, lo que deja a su gente a la deriva de procesos sociales aun más complejos, como la redistribución y resignificación de poder en las sociedades capitalistas actuales.

Aunque no tengamos evidencia en este estudio para vincular directamente los grandes procesos de rearticulación de poder que viven nuestras sociedades actuales, cuando un agente cultural es invitado a participar del Programa ellos perciben que ocupan otro lugar. En consecuencia, reciben otro trato por parte de sus vecinos o su comunidad.

"Desde que empecé con este trabajo de las plantitas, porque es un trabajo, imagínese usted que ya no hay plantas que antes había acá, -yo rescato eso- a mí la gente me saluda y siempre me andan preguntando cositas. Incluso si no tienen plata yo los atiendo igual y les doy recetas caseras $y$ a veces me pagan con unos kilos de carne o otra cosa. Es ¿cómo le dijera yo a usted? Es como trueque (intercambio), ¿vio?" (lawentuchefe, sector Pocura).

Es evidente que la percepción sobre la relación entre persona y naturaleza para los entrevistados refleja una ontología de la naturaleza o perspectiva fundamental de orden, en la cual el centro no son las personas, sino el ambiente. En ese sentido, emerge en el discurso una percepción de conservación y protección, ya que para ellos es impensable de otra manera (Tabla 1). Por lo tanto, la necesidad de contar con alguien que conozca de medicina mapuche es alta y recurrente en los espacios rurales de Panguipulli. Buscar algo de sabiduría local ancestral es común $\mathrm{y}$ fundamental entre sus habitantes y, por lo tanto, quien posea ese conocimiento es muy respetado.

La tradición mapuche considera a la persona como gente de la tierra, le pertenece a ella (mapu: tierra, che: gente). Sobre esta perspectiva, los entrevistados recordaban 
cómo les habían enseñado desde su temprana infancia a hablar con las plantas si es que se necesitaba cortar una hoja. También reconocen un poder especial en la naturaleza, ya que "bastaba caminar en el bosque para recargar energías u olvidar las penas" (extracto del relato de la lawentuchede del sector de Pocura, Panguipulli). En este sentido, la percepción del medio ambiente y por añadidura, de los PMNL, también aparece como biocéntrica.

Finalmente, la percepción en términos culturales que fue posible evidenciar a través de las historias de vida es la constante preocupación por rescatar la sabiduría ancestral no sólo en lo referente a los PMNL, sino también conocimiento vinculado a los ciclos naturales que rigen la existencia de la vida según la cosmovisión mapuche. En este sentido, las entrevistas corroboran la sensación de pérdida de plantas y de personas que conocen o tienen la autoridad para sanar a través de la medicina natural.

El caso más emblemático, citado de forma recurrente en las historias de vida, es el de las parteras, literalmente ocultas por una serie de razones. Son historias que van desde persecuciones de la época de la dictadura militar chilena hasta el miedo a ser arrestadas el día de hoy por considerarse prácticas poco higiénicas por parte del Estado chileno. No obstante, la figura de la partera es muy respetada por su acabado conocimiento de hierbas útiles para tratar a varios tipos de seres vivientes, llegando a ser percibida a veces incluso como enciclopédica.

"Imagínese que ella conocía plantas para tratar animales y personas. Como el tema de ella era el embarazo, las personas somos igual que las plantas y los animales, funcionamos con la luna de cosecha (llena) y la de siembra (nueva). Entonces te podía ayudar a quedar embarazada, a elegir si querías niña o niño, cosas así. También podía hacer tratamientos para animales, para que tuvieran de a dos o incluso más (...)" (ñaña, sector Pullingue).

Tanto para las personas como para los animales, la presencia de la partera era central, lo que deja en claro que el proceso de nacer es lo suficientemente complejo como para la necesidad de su asistencia. Esta percepción negativa se sustenta entonces en la pérdida concreta de prácticas y saberes que se dejan de necesitar y demandar. Esto nos lleva a pensar que si la necesidad se mantiene, mientras que el sistema o estrategia de satisfacción desaparece existiría algún nivel de anomia social o reemplazo de orden normativo por otro distinto, asociado, en este caso, a las prácticas sociales vinculadas al proceso de construcción de bienestar local.

Los satisfactores como estrategias para alcanzar las necesidades humanas a partir del uso de los PMNL. Las nueve necesidades humanas fundamentales, por ser universales, son de fácil reconocimiento por los entrevistados. En ese sentido, la identificación

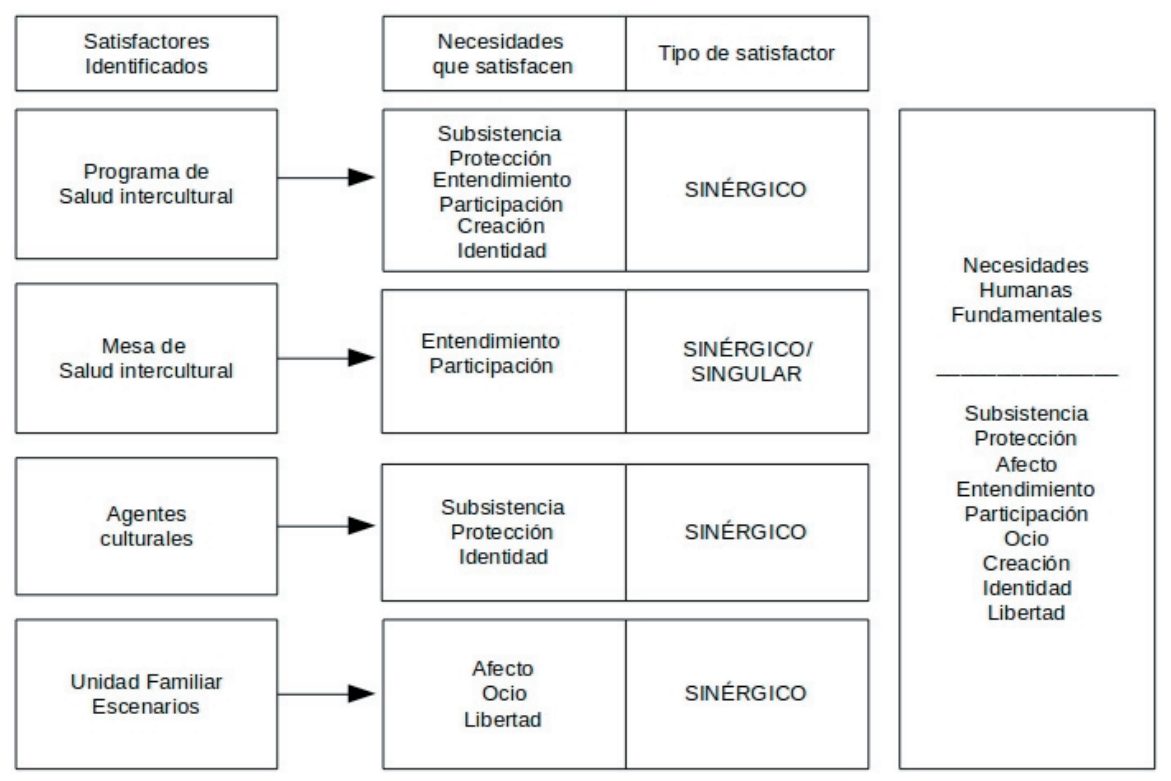

Figura 3. Satisfactores asociados al uso de PMNL en Panguipulli. Cada satisfactor se relaciona directamente con al menos dos necesidades humanas fundamentales.

Figure 3. Satisfiers linked to the use of natural medicine products in Panguipulli municipality. Each satisfier is directly related to at least two basic human needs. 
de los satisfactores o de las estrategias de satisfacción por parte de los entrevistados fue un trabajo de análisis posterior a los relatos. Los satisfactores asociados al uso de PMNL se dividieron en cuatro de acuerdo al impacto declarado por los entrevistados.

En este sentido, el más importante fue el mismo Programa, ya que, como estrategia, es posiblemente un satisfactor sinérgico (Figura 3). Con su operatividad, aporta a satisfacer varias necesidades humanas fundamentales, según los entrevistados, y no sólo aporta a rescatar la identidad local a través de la búsqueda de agentes culturales, sino que al conseguirlo ayuda a satisfacer la necesidad de subsistencia, protección, entendimiento, participación y creación de quienes se relacionan con esta iniciativa, ya sea entregando küme lawen o recibiéndolo.

Los segundos en importancia son la Mesa de Salud Inter-Cultural y los agentes culturales. Ambos están contenidos como satisfactores dentro del satisfactor sinérgico que representa el Programa. Sin embargo, son estrategias totalmente distintas ya que hay Mesas repartidas porel territorioy apuntan a satisfacer las necesidades particulares de entendimiento y participación de cada localidad donde se encuentran. Esto es un incentivo para que la comunidad participe e identifique agentes culturales. Al mismo tiempo, con su presencia, los agentes culturales refuerzan la estrategia de satisfacción para alcanzar subsistencia, protección e identidad al participar de su comunidad con la impronta que les entrega su participación en el Programa.

Además, la unidad familiar se erige como un satisfactor reconocible en las historias de vida y especialmente relacionado con la construcción de bienestar al cumplir con la función esencial de dar vida a prácticas cotidianas de recolección de plantas medicinales y la aplicación y enseñanza de técnicas concretas para tratamientos de salud no tan complejos. Aquí, las prácticas más comunes son la relación con el cultivo, la recolección, el secado, la prescripción de infusiones, los macerados, los ungüentos y las compresas. Prácticas idénticas a las del Programa, con la única diferencia que en éste se trabaja de manera comunitaria o pública, se trabajan en la familia de manera íntima o privada. No obstante, la unidad familiar como satisfactor apunta hacia las únicas necesidades que no fue posible ver desde el Programa: el afecto, el ocio y la libertad. La necesidad de afecto es la que muchas veces gatilla el uso de un PMNL dentro de la familia, de tal forma que el uso de este SE satisface la necesidad de compartir y enseñar en momentos de ocio. Entonces, la transmisión entre generaciones o pares encuentra un lugar de reproducción.

"Ella (hija mayor) se fue a Santiago, pero aquí está mi retoño, la más chica (6 años) y yo la quiero mucho y la cuido mucho. Me preocupo harto de ella. Siempre le explico cada cosita que le doy. Ella es bien interesada, como quien dice... busquilla (curiosa) y cuando me ve sacando algo del jardín o del bosque le llama mucho la atención. Ya sé que ella podría tomar mi lugar si es que le interesa. Yo creo que sí" (lawentuchefe, sector Pocura).

Los escenarios donde se emplaza la unidad familiar contienen y componen la estrategia que les permite apuntar a satisfacer la necesidad de libertad. En los relatos fue recurrente el comentario "vamos a buscar pastitos", actividad de recolección estrictamente medicinal, de reconocimiento tipo inventario, y no en pocas ocasiones celada por el veto del secreto. No obstante, les permitía entre otras cosas, recordar y reafirmar lo que saben, junto con dar vida a memorias de infancia y experimentar la "sensación de energía que entrega el bosque". Los trayectos recorridos, en su mayoría escenarios compuestos por bosque nativo del sur de Chile, orillas de ríos, lagos, cerros y cascadas, reafirman para los entrevistados la necesidad de sentirse libres.

"Mire, yo he vivido por estos lados siempre. Me crié aquí. He pasado de todo, en serio, de todo. Hasta hijos tuve en este campo... al aire libre ise imagina? (risas). Bueno, como le decía, si me bajoneo (entristezco) o tengo algún problema, yo prefiero hacer este mismo recorrido hasta la cascada. No sé si eso es ser libre, pero me ayuda-como quien dice-me reconforta, hasta el momento nadie me lo ha podio quitar" (lawentuchefe, sector Liquiñe).

\section{Discusión y CONCLUSIONES}

Las historias de vida demuestran que los PMNL se relacionan potencialmente con diversos componentes del bienestar. El más claro servicio/beneficio es el uso medicinal, pero también existen otros como tradiciones familiares (en el caso del pueblo mapuche), cultura popular y órdenes económicos no crematísticos locales, que de acuerdo con los relatos y las prácticas convocan buenas relaciones sociales y acceso a bienes materiales para una buena vida (MEA 2005). En este 
sentido, las personas entrevistadas construyen bienestar socialmente a través de prácticas cotidianas dentro de la unidad familiar, en un ambiente rutinario y privado.

Por otra parte, también se construye bienestar en otro nivel a través de prácticas de índole comunitaria o pública. Es entre lo público y lo privado que, justamente, las prácticas sociales asociadas a los PMNL obtienen la dinámica necesaria para dar vida a un tránsito que aporta al bienestar, de manera local, con al menos cuatro satisfactores reconocibles en este territorio. Cada uno con sus componentes y aportes claros hacia las necesidades humanas o hacia alguno de los constituyentes del bienestar según MEA (Figura 3).

Una de las características más relevantes de las prácticas asociadas al uso de PMNL es que se vinculan con una batería de percepciones con carga positiva y negativa (Tabla 1). La relación entre PMNL como servicio y bienestar es una relación compleja ya que se sostiene a través del trabajo mutuo entre personas y naturaleza, lo que genera más de un trayecto proveedor de beneficios, situación que se vuelve posible tanto por iniciativas individuales y grupales. También porque diversas dimensiones del SE aportan a distintos constituyentes del bienestar y a través de distintos satisfactores. Desde el aprovisionamiento las prácticas sociales se remiten a procesar y regenerar el conocimiento ancestral. Al mismo tiempo, las prácticas sociales inevitablemente definen y redefinen tanto al habitus como al campo social. En ese sentido, la dimensión político-cultural del SE emerge con claridad. Los resultados corroboran la reciente idea de coproducción de SE, propuesta desde la literatura. Entre otras cosas, esta propuesta reconoce que los servicios/beneficios que la gente obtiene de los ecosistemas para su bienestar no son producidos independientemente de los seres humanos, sino que surgen de las interacciones entre las personas con un ecosistema (Comberti et al. 2015; Fisher et al. 2016). Sin embargo, estas relaciones han sido poco investigadas. Conocer el habitus y definir su campo social permite identificar características básicas de la disposición a actuar y de las prácticas sociales en las que incurren los usuarios del SE. En términos sociológicos, sería estudiar la perspectiva fundamental a través de la cual los agentes culturales ordenan la realidad y ejercen sus prácticas, lo que permite desarrollar una relación particular entre persona y naturaleza a través del uso local de este SE.

El SE PMNL es muy sensible a liderazgos específicos $\mathrm{y}$, en particular, está expuesto a un escenario social adverso porque no hay sólo una sociedad en este territorio, hay más orígenes fundamentales, 'entendiendo' la realidad y la naturaleza. Si bien el CESFAM de Panguipuli es una institución formal del Estado chileno, el Programa de Medicina Intercultural responde a una necesidad de pueblos indígenas. Desde esta perspectiva emergen las características provenientes de la carga negativa de las percepciones de los entrevistados: ellos sugieren una situación intensa de pérdida del SE en cuanto a magnitud (cada vez menor stock en los ecosistemas) y a calidad (cada vez menos conocimientos).

Los satisfactores revelados por la investigación surgen como una estrategia socialmente construida para ocupar espacio en el campo social de la salud, del uso del paisaje y del ambiente. De esta forma, en su dimensión política, el Programa de Salud Inter-Cultural es, justamente, una estrategia que busca posicionar y representar una determinada política del uso de la naturaleza, en función de mantener o tratar los diversos estados de salud que presentan las personas a lo largo de su vida. En ese sentido, los satisfactores reconocidos apuntan a una administración social del SE en función de la salud, y de una forma puntual de interpretar (y relacionarse con) la naturaleza. Esto nos lleva a evaluar que la construcción de bienestar por parte de las comunidades locales en Panguipulli pasa por un proceso de rescate, reconstrucción casi arqueológica del saber sobre el uso tradicional del entorno natural, prácticas y conocimiento sobre el uso del SE. La disposición social a actuar de parte de los entrevistados los inclina a practicar un uso del lawen, de la medicina natural, de manera rutinaria a lo largo de sus vidas para mantener la salud y el entorno, y no para tener que rescatarlos.

La percepción de pérdida de algunas plantas (es decir, que "ya no las han visto más por ahí", típica expresión de los agentes culturales para explicar que cada vez encuentran menos lawen [plantas]), junto con la falta de agentes culturales 'sabios' intensifica el habitus o la disposición a cultivar, cuidar o rescatar para lograr llegar al óptimo (que sería una vida en equilibrio con el entorno natural). Siguiendo en la línea de la dimensión política, como parte de la lucha de posicionamiento, el fenómeno de resignificar rol y estatus de personas de la comunidad que se vuelven agentes culturales del Programa pasa por el re-conocimiento de su sabiduría sobre el uso de PMNL y, en particular, por su uso 
en práctica medicinal. Esta es una hábil estrategia de posicionamiento político para darle operatividad a la práctica médica local y a la provisión del SE. Aquí es donde el SE promueve un espacio de significación política ya que lo administran las comunidades locales desde su posicionamiento cultural, social y médico. Al mismo tiempo que re-significa el rol y el estatus de personas en las comunidades locales, el Programa usa el SE y construye bienestar al mantenerse funcionando.

Por su parte, el discurso del küme mongen, que aboga por un vivir bien, es una idea recurrente en los cuatro satisfactores identificados en esta exploración: a) Programa; b) Mesa de Salud Inter-Cultural; c) agentes culturales, y d) unidades familiares. Los cuatro satisfactores como estrategias de administración oikonómicas, apuntan a construir bienestar desde una impronta política diferenciada. La identificación del habitus en las prácticas de producción de la naturaleza desde la lógica de la medicina mapuche muestran una disposición a actuar en el campo social de la medicina y del uso del bosque, que advierten el sentido práctico de esta acción social: rescatar conocimiento, significar y posicionar roles en el campo social. Esta situación ilumina el espacio de la construcción de bienestar como un territorio de posiciones de poder. La dimensión política de los PMNL en Panguipulli es un área que refleja un importante capital cultural hábilmente desplegado a través de los satisfactores identificados.

En esencia, una de nuestras conclusiones apunta a rebatir la visión muchas veces unidireccional entre SE y bienestar como la expresada en el marco de MEA (2005) o en el modelo de cascada (Haines-Young and
Potschin 2010), en favor de relaciones mucho más complejas (Fischer and Eastwood 2016). Lo primero es que un SE como este no puede ser definido a priori como de provisión o cultural, sino que lo definen las personas a través de sus valores, percepciones, usos, entre otros. En segundo lugar, los SE son sinérgicos al desarrollo de otros satisfactores que en conjunto satisfacen necesidades humanas fundamentales y componentes del bienestar. En ese sentido, si desaparecen las personas con el conocimiento sobre el uso, ¿desaparece el SE en su dimensión cultural? Si se modifican las prácticas y las costumbres, ¿seguimos hablando del mismo SE? Mirar el vínculo entre un SE cultural y el proceso de construir bienestar, englobados para estas comunidades bajo el concepto de küme lawen y/o el discurso de küme mongen del pueblo mapuche, es mirar una estrategia social de aprovechamiento, producción o co-producción de la naturaleza. esto implica explorar la manera en que cualitativamente se valora a la naturaleza. En este sentido, la gestión del territorio y del uso de los PMNL por estas comunidades está acompañada de un proceso de legitimación social de actores, proyectos de investigación, conflictos ontológicos, epistemológicos y posiciones de poder diferentes y divergentes.

AgradeCIMIENTOS. Este trabajo fue patrocinado por los proyectos FONDECYT N ${ }^{\circ} 1151187$ e IAI CRN3 095 (con la ayuda de una subvención del Instituto Interamericano por el Cambio Global, con apoyo de la Fundación Nacional de Ciencias de Estados Unidos, Grant GEO1128040). Agradecemos especialmente el apoyo de la organización Bosque Modelo Panguipulli y del Programa de Salud InterCultural de Panguipulli.

\section{REFERENCIAS}

Ardila, C., A. Biersack, S. Castro-Gómez, P. Descola, E. Dussel, A. Escobar, et al. 2011. Cultura y Naturaleza. L. Montenegro, Ed. Jardín Botánico de Bogotá José Celestino Mutis. https://goo.gl/BFSKoG.

Balvanera P., M. Uriarte, L. Almeida-Leñero, A. Altesor, F. De Clerck, T. Gardner, J. Hall, A. Lara, P. Laterra, M. PeñaClaros, D. Silva, L. Romero-Duque, A. Vogl, L. Arreola, A. Caro-Borrero, F. Gallego, M. Jain, Ch. Little, R. Olvieira, J. Paruelo, J. Peinado, L. Poorter, N. Ascarrunz, F. Correa, M. Cunha-Santino, A. Hernández-Sánchez, and M. Vallejos. 2012. Ecosystem services research in Latin America: The state of the art. Ecosystem Services 2:56-70.

Bennet, E., W. Cramer, A. Begossi, G. Cundill, S. Díaz, B. Egoh, I. Geizendorffer, C. Krug, S. Lavorel, E. Lazos, L. Lebel, B. Martín-López, P. Meyfroidt, H. Mooney, J. Nel, U. Pascual, K. Payet, N. Harguindeguy, G. Peterson, A. PreiurRichard, B. Reyers, P. Roebeling, R. Seppelt, M. Solan, P. Tschakert, T. Tscharntke, B. Turner, P. Verburg, E. Viglizzo, P. White, and G. Woodward. 2015. Linking biodiversity,ecosystem services, and human well-being: three challenges for designing research for sustainability. Current Opinion in Environmental Sustainability 14:76-85.

Bourdieu, P. 1977. Outline of A Theory of Practice. Cambridge, Cambridge University Press.

Bourdieu, P., and L. Wacquant. 2005. Una invitación a la sociología reflexiva. Siglo XXI Editores.

Butler, C. D., and W. Oluoch-kosura. 2006. Linking Future Ecosystem Services and Future Human Well-being, Ecology and Society 11(1), art. 30. https://goo.gl/IHPzds.

Daw, T., K. Brown, S. Rosendo, R. Pomeroy. 2011. Applying the ecosystem services concept to poverty alleviation: the need to disaggregate human well-being. Environ Conserv 38:370-379. 
Carpenter, S. R., H. A. Mooney, J. Agard, D. Capistrano, et al. 2009. Science for managing ecosystem services: Beyond the Millennium Ecosystem Assessment. Proceedings of National Academy of Sciences 106(5):1305-1312. https: L/goo.gl/QMVoUK.

Church, A., J. Burgess, N. Ravenscroft, K. Blackstock, E. Brady, et al. 2011. Cultural Services. UK National Ecosystem Assessment: Technical Report 633-692. https://goo.gl/6cVSCS.

Comberti, C., et al. 2015. Ecosystem services or services to ecosystems? Valuing cultivation and reciprocal relationship between humans and ecosystems. global Environmental Change 34:247-262.

Fish, R., J. Burgess, A. Church, and K. Turner. 2011. Chapter 24: Shared Values for the Contributions Ecosystem Services Make to Human. UK National Ecosystem Assessment: Technical Report, 1183-1194. https://goo.gl/IrGktj.

Fischer, A., and A. Eastwood. 2015. Coproduction of ecosystem services as human-nature interactions - An analytical framework. Land Use Policy 52: 41-50. March 2016. ISSN 0264-8377. http://dx.doi.org/10.1016/

Geertz, C. 1996. La interpretación de las culturas. Barcelona: Gedisa.

Guerry, A., S. Polasky, J. Lubchenco, R. Chaplin-Kramer, G. Daily, R. Griffin, M. Ruckelshaus, I. Bateman, A. Duraiappah, T. Elmqvist, M. Feldman, C. Folke, J. Hoekstra, P. Kareiva, B. Keeler, S. Li, E. McKenzie, Z. Ouyang, B. Reyers, T. Ricketts, J. Rockström, H. Tallis, and B. Vira. 2015. Natural capital and ecosystem services informing decisions: From promise to practice. PNAS 212(24):7348-7345.

Sandhu, H., and S. Sandhu. 2014. Linking ecosystem services with the constituents of human well-being for poverty alleviation in eastern Himalayas. Ecological Economics 107:65-75.

Instituto Nacional de Estadísticas (INE). 2003. Censo 2002: Síntesis de resultados. https:/ /goo.gl/ejN1wS.

Iniesta-Arandia, I., M. García-Llorente, P. Aguilera, C. Montes, and B. Martín-López. 2014. Socio-cultural valuation of ecosystem services: uncovering the links between values, drivers of change, and human well-being. Ecological Economics 108:36-48.

Jackson, M. 1983. Knowledge and the Body. Man 18(2):327-45.

Kull, C. A., X. Arnauld de Sartre, and M. Castro-Larrañaga. 2015. The political ecology of ecosystem services. Geoforum 61:122-134. http://doi.org/10.1016/j.geoforum.2015.03.004

Max-Neef, M., A. Elizalde, and M. Hopenhayn. 1993. Desarrollo a Escala Humana. Editorial Nordan-Comunidad e Icaria Editorial. https://goo.gl/8itMX3.

Millennium Ecosystem Assessment. 2005. Ecosystems and human Well-being: Synthesis.

Miranda, A., A. Altamirano, L. Cayuela, F. Pincheira, and A. Lara. 2015. Different times, same story:Native forest loss and landscape homogenization in three physiographical areas of south central of Chile. Applied Geography 60: 20-28 http://doi.org/10.1016/j.apgeog.2015.02.016.

Montañés, M. 2013. Diseño científico de muestras estructurales. Dialnet. Actas Del 2do Congreso Nacional Sobre Metodología de La Investigación. Comunicación 4. Vol. 4. https://goo.gl/jk9UJ2.

Nahuelhual L., P. Laterra, S. Villarino, M. Mastrangelo, A. Carmona, A. Jaramillo, P. Barral, and N. Burgos. 2015. Mapping of ecosystem services: missing links between purposes and procedures, Ecosystem Services 13:162-172.

Ostrom, E. 2009. A general framework for analyzing sustainability of social-ecological systems. Science 325(5939): 419-22. http://doi.org/10.1126/science. 1172133

Polasky, S., and K. Segerson. 2009. Integrating ecology and economics in the study of ecosystem services: some lessons learned. Annual Review of Resource Economics 1:409-434. doi:10.1146/annurev.resource.050708.144110.

Skewes, J. C., D. Guerra, P. Rojas, and A. Mellado. 2011. ¿La memoria de los paisajes o los paisajes de la memoria? Desenvolvimiento E Meio Ambiente 23:39-57.

Suich, H., C. Howea, and G. Mace. 2015. Ecosystem services and poverty alleviation: A review of the empirical links. Ecosystem Services 12:137-147.

Xu, H., H. Zheng, X. Chen, Y. Ren, and Z. Ouyang. 2016. Relationships between river water quality and landscape factors in Haihe River Basin, China: implications for environmental management. Chinese Geographical Science 26(2):197-207. doi: 10.1007/s11769-016-0799-9. 